\title{
On the Tribonacci fractals
}

\author{
T. Sellami \\ Dept. of Mathematics, Faculty of sciences of Sfax, Sfax University, Sfax, Tunisia \\ Available online at: www.isroset.org \\ Received 26/Jan/2018, Revised 15/Feb/2018, Accepted 17/Mar/2018, Online 30/Apr/2018
}

\begin{abstract}
We consider the two tribonacci substitutions, $\mathrm{a} \rightarrow \mathrm{ab}, \mathrm{b} \rightarrow \mathrm{ac}, \mathrm{c} \rightarrow \mathrm{a}$ and $\mathrm{a} \rightarrow \mathrm{ab}, \mathrm{b} \rightarrow \mathrm{ca}, \mathrm{c} \rightarrow \mathrm{a}$. In this paper we give some properties for the Tribonacci fractals, and its associated intersection fractal. We show in more details intersections of subtiles.
\end{abstract}

Keywords—Rauzy fractals, Substitution dynamical systems, balanced pairs algorithm.

\section{INTRODUCTION}

Rauzy considered as a generalisation of the Fibonacci substitution the substitution $\sigma: \mathrm{a} \rightarrow \mathrm{ab}, \mathrm{b} \rightarrow \mathrm{ac}, \mathrm{a} \rightarrow \mathrm{a}$ over the alphabet $\{a, b, c\}$. Starting with a and repeatedly applying $\sigma$ we get successively $u_{\mathrm{n}}=$ abacabaabacababac... . The limit word is called the Tribonacci word. Note that if we define $T_{0}=0, T_{1}=T_{2}=1, T_{\mathrm{n}}=T_{\mathrm{n}-1}+T_{\mathrm{n}-2}+T_{\mathrm{n}-3}$ for $\mathrm{n}>2$, then the number of symbols pf $u_{\mathrm{n}}$ equals $T_{\mathrm{n}+2},\left|u_{\mathrm{n}}\right|_{\mathrm{a}}=T_{\mathrm{n}+1}$,

$$
\left|u_{\mathrm{n}}\right|_{\mathrm{b}}=T_{\mathrm{n}},\left|u_{\mathrm{n}}\right|_{\mathrm{c}}=T_{\mathrm{n}-1} \text {. }
$$

The ferquency of a equals $\lim _{n} \rightarrow \infty \frac{T \mathrm{n}+1}{T \mathrm{n}+2}=\tau$, the ferquency of b equals $\lim _{n} \rightarrow \infty \frac{T \mathrm{n}}{T \mathrm{n}+2}=\tau^{2}$, the ferquency of $c$ equals $\lim _{n} \rightarrow \infty \frac{T \mathrm{n}-1}{T \mathrm{n}+2}=\tau^{3}$, where $\tau+\tau^{2}+\tau^{3}=1$. Since the frequencies are irrational, the Tribonacci sequence is nonperiodic.

The Tribonacci word is not balanced with respect to each letter. The wod $\mathrm{u}_{5}$ contains subwords of length 3 without 1 and a subwords of length 3 with two 1's.

The Tribonacci word is episturmian on three letters which implies that $P(\mathrm{n})=2 \mathrm{n}+1$ for every $\mathrm{n}$. Because a word on three letters is periodic if the frequencies of its letters are independent over $\boldsymbol{Q}$.

The Tribonacci word can be described as a cutting sequence. In fact, in the $\mathrm{x}, \mathrm{y}, \mathrm{z}$ space broken Tribonacci halfine that we get by starting in $(0,0,0)$ and going 1 in the direction of the $\mathrm{y}$-axis if $t_{m}=\mathrm{a}$, in the direction of the y-axis if $t_{m}=\mathrm{b}$, in the direction of the $\mathrm{z}$-axis if $t_{m}=c$ provides an excellent discrete approximation.

$$
\mathrm{c} \rightarrow \mathrm{a} \quad \mathrm{c} \rightarrow \mathrm{a}
$$

The incidence matrix of $\sigma_{1}$ and $\sigma_{2}$ is

$M \sigma=\left(\begin{array}{ccc}1 & 1 & 1 \\ 1 & 0 & 0 \\ 0 & 1 & 0\end{array}\right)$. By induction we find that

The dominant eigenvalue satisfies the relation $X^{3}-X^{2}-X^{1}=0$, hence that

$\mathrm{M}_{\sigma}{ }^{\mathrm{n}}=$

$$
\left(\begin{array}{ccc} 
& & \\
\mathrm{Tn}+1 & \mathrm{Tn} & \mathrm{Tn}-1 \\
\mathrm{Tn}+\mathrm{Tn}-1 & \mathrm{Tn}-1+\mathrm{Tn}-2 & \mathrm{Tn}-2+\mathrm{Tn}-3 \\
\mathrm{Tn} & \mathrm{Tn}-1 & \mathrm{Tn}-2
\end{array}\right)
$$

name Tribonacci for the substitution.

It follows that for $\mathrm{n} \geq 2$ the word $u_{\mathrm{n}}=\sigma^{\mathrm{n}-2}\left(u_{2}\right)$ originates from the word $u_{2}=$ abac where $u_{n}$ the first letters come from the first a in $u_{2}$, the next $T_{\mathrm{n}-1}+T_{\mathrm{n}-2}$ letters come from $\mathrm{b}$, the next $T_{n}$ letters have their origin in the second a and the last $T_{\mathrm{n}-1}$ letters are generated by $c$. The total number is $T_{\mathrm{n}-2}$ is indeed.

Although the fixed point of each substitutions have dynamical and geometrical properties.

The Rauzy fractal of the first substitution is a topological disc [1], simply connected, while it is a well known fact that the second fractal is not simply connected, compare Figure[1].

Let $\sigma_{1}$ and $\sigma_{2}$ be the two Tribonacci substitutions :

$$
\sigma_{1}:\left\{\begin{array}{l}
\mathrm{a} \rightarrow \mathrm{ab} \\
\mathrm{b} \rightarrow \mathrm{ac}
\end{array} \quad \text { and } \quad \sigma_{2}:\left\{\begin{array}{l}
\mathrm{a} \rightarrow \mathrm{ab} \\
\mathrm{b} \rightarrow \mathrm{ca}
\end{array}\right.\right.
$$




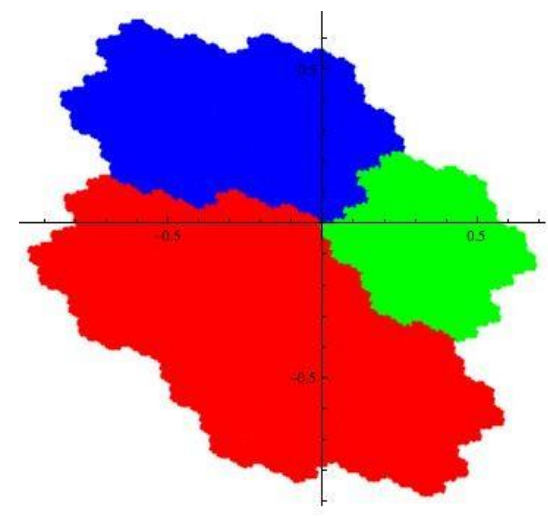

Figure [1] : the Rauzy fractal of $\sigma_{1}$.

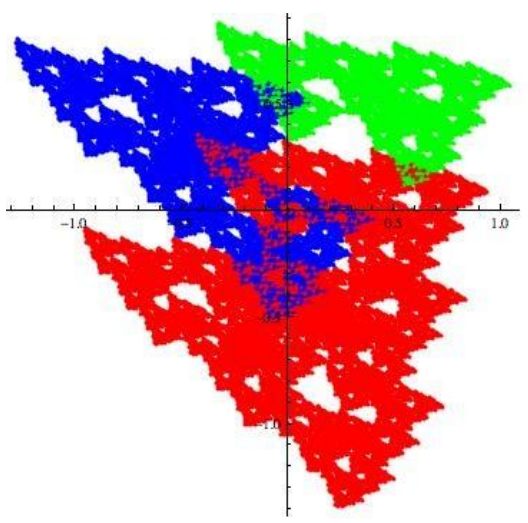

Figure [2] : the Rauzy fractal of $\sigma_{2}$.

In this paper we will studies the common points of the two stepped lines associated to $\sigma_{1}$ and $\sigma_{2}$ and we will proof that these common points can be generated by a morphisim on the so called balanced pair.

\section{SUbSTITUTIONS AND RAUZY FRACTALS}

\section{A. General setting}

Let $\boldsymbol{A}:=\left\{\mathrm{a}_{1}, \ldots, \mathrm{a}_{\mathrm{d}}\right\}$ be a finite set of cardinal d called alphabet. The free monoid $\boldsymbol{A}^{*}$ on the alphabet $\boldsymbol{A}$ with empty word $\varepsilon$ is defined as the set of finite words on the alphabet $\boldsymbol{A}$, this is $\boldsymbol{A}^{*}:=\mathrm{U}_{\mathrm{k} \in \mathbb{N}} \boldsymbol{A}^{\mathrm{k}}$, endowed with the concatenation map. We denote by $\boldsymbol{A}^{\mathbb{N}}$ and $\boldsymbol{A}^{\mathbb{Z}}$ the set of one and two-sided sequences on $\boldsymbol{A}$, respectively. The topology of $\boldsymbol{A}^{\mathbb{N}}$ and $\boldsymbol{A}^{\mathbb{Z}}$ is the product topology of discrete topology on each copy of $\boldsymbol{A}$. Both spaces are metrizable.

The length of a word $\mathrm{w} \epsilon \boldsymbol{A}^{\mathrm{n}}$ with $\mathrm{n} \epsilon \mathrm{N}$ is defined as $|w|=n$. For any letter $a \in A$, we define the number of occurrences of a in $\mathrm{w}=\mathrm{w}_{1} \mathrm{w}_{2} \ldots \mathrm{w}_{\mathrm{n}-1} \mathrm{w}_{\mathrm{n}}$ by $|\mathrm{w}|_{\mathrm{a}}=\#\left\{\mathrm{i} \mid \mathrm{w}_{\mathrm{i}}=\mathrm{a}\right\}$.

Let $1: A^{*} \rightarrow Z^{\mathrm{d}}: \mathrm{w} \mapsto\left(|\mathrm{w}|_{\mathrm{a}}\right)_{\mathrm{ac} A} \in \mathrm{N}^{\mathrm{d}}$ be the natural homomorphism obtained by abelianization of the free monoid, called the abelianization map. A substitution over the alphabet $\boldsymbol{A}$ is an endomorphism of the free monoid $\boldsymbol{A}$ such that the image of each letter of $\boldsymbol{A}$ is a nonempty word.

A substitution $\sigma$ is primitive if there exists an integer $\mathrm{k}$ such that, for each pair $(\mathrm{a}, \mathrm{b}) \in \boldsymbol{A}^{2},\left.|| \operatorname{sigma}^{\mathrm{k}}(\mathrm{a})\right|_{\mathrm{b}}>0$. We will always suppose that the substitution is primitive, this implies that for all letter $\mathrm{j} \in \boldsymbol{A}$ the length of the successive iterations $\sigma^{\mathrm{k}}(\mathrm{j})$ tends to infinity.

A substitution naturally extends to the set of two sided sequences $\boldsymbol{A}^{\mathbb{Z}}$. We associate to every substitution $\sigma$ its incidence matrix $\mathrm{M}$ which is the $\mathrm{n} \times \mathrm{n}$ matrix obtained by abelianization, i.e. $\mathrm{M}_{\{\mathrm{i}, \mathrm{j}\}}=|\sigma(\mathrm{j})|_{\mathrm{i}}$. It holds that $\mathrm{l}(\sigma(\mathrm{w}))=\mathrm{Ml}(\mathrm{w})$ for all $\mathrm{w} \in \boldsymbol{A}^{*}$.

Remark. The incidence matrix of a primitive substitution is a primitive matrix, so with the Perron-Frobenius theorem, it has a simple real positive dominant eigenvalue $\beta$.

\section{B. Rauzy fractals}

Definition 2.1. A Pisot number is an algebric integer $\beta>1$ such that each Galoisconjugate $\beta^{(\mathrm{i})}$ of $\beta$ satisfies $\left|\beta^{(\mathrm{i})}\right|<1$.

From now, we will suppose that all the substitutions that we consider are irreducible of Pisot type and unimodular. This mean that the characteristic polynomial of its incidence matrix is irreducible, its determinant is equal to \pm 1 and its dominant eigenvalues is a Pisot number. We can prove that any irreducible Pisot substitution is primitive (see [11, 5, 6, 7]).

Remark. Note that there exist substitution whose largest eigenvalue is Pisot but whose incidence matrix has eigenvalues that are not conjugate to the dominant eigenvalue. Example is $1 \rightarrow 12,2 \rightarrow 3$,

$3 \rightarrow 4,4 \rightarrow 5,5 \rightarrow 1$. The characteristic

polynomial is reducible. Such substitutions are called Pisot reducible.

Definition 2.2. Let $\sigma$ a substitution and $u \in \boldsymbol{A}^{N}, u$ is a fixed point of $\sigma$ if $\sigma(u)=u$. The infinite word $\mathrm{u}$ is a periodic point of $\sigma$ if there exist $\mathrm{k} \in \mathrm{N}$ such that $\sigma^{\mathrm{k}}(u)=u$.

Let $\sigma$ be a primitive substitution, then there exist a finite number of periodic points (see [10]). We associate to the fixed point $u$ of the substitution a symbolic dynamical system $\$\left(\Omega_{u}, S\right)$ where $S$ is the shift map on $\boldsymbol{A}^{\mathbb{N}}$ given by $S\left(\mathrm{a}_{0}\right.$ $\left.\mathrm{a}_{1} \ldots\right)=\mathrm{a}_{1} \mathrm{a}_{2} \ldots$ and $\Omega_{u}$ is the closure of $\left\{S^{\mathrm{n}}(\mathrm{u}): \mathrm{m} \geq 0\right\}$ in $A^{N}$.

Remark. If $\sigma$ is a primitive substitution then the symbolic 
dynamical system $(\Omega u, S)$ does not depend on $u$; we denotetite $\mathrm{X}_{\sigma}$ are naturally defined, depending on the letter by $\left(\Omega_{\sigma}, S\right)$.

We say that a dynamical system $(\mathrm{X}, \mathrm{f})$ is semiconjugate to another dynamical system (Y, g) if there exists a continuous surjective

map $: \mathrm{X} \rightarrow \mathrm{Y}$ such that $\theta^{\circ} \mathrm{f}=\mathrm{g}$ o $\theta$ An important question is whether and how the symbolic dynamical system $\left(\Omega_{u}, S\right)$ admit ageometric model. By geometrically realizable we mean there exists a dynamical

system $(\mathrm{X}, \mathrm{f})$ defined on a geometrical structure, such that $\left(\Omega_{u}, S\right)$ is semiconjugate to $(\mathrm{X}, \mathrm{f})$.

In [13], G.Rauzy proves that the dynamical system generated by the substitution $\sigma(1)=12, \sigma(2)=13, \sigma(3)=1$, is measuretheoretically conjugate to an exchange of domains in a compact set $R$ of the complex plane. This compact subset has a self-similar structure : using method introduced by F.M.Dekking in [9], S.Ito and Pierre Arnoux obtain in [1] an alternative construction of $R$ and prove that each of exchanged domains has fractal boundary. We will use the projection method to obtain the Rauzy fractal.

Definiton 2.3. A stepped line $\mathrm{L}=\left(\mathrm{x}_{\mathrm{n}}\right)$ in $\underline{\mathrm{R}}^{\mathrm{d}}$ is a sequence (it could be finite or infinite) of points in $\mathbb{R}^{\mathrm{d}}$

such that $\mathrm{x}_{\mathrm{n}+1}-\mathrm{X}_{\mathrm{n}}$ belong to a finite set.

A canonical stepped line is a stepped line such that $\mathrm{x}_{0}=0$ and for all $n \geq 0, x_{n+1}-x_{n}$ belong to the canonical basis of $\mathbb{R}^{d}$.

Using the abelianization map, to any finite or infinite word $\mathrm{W}$, we can associate a canonical stepped line in $\mathbb{R}^{\mathrm{d}}$ as a sequence $\left(1\left(P_{\mathrm{n}}\right)\right)$, where $P_{\mathrm{n}}$ are the prefix of length $\mathrm{n}$ of $\mathrm{W}$.

An interesting property of the canonical stepped line associated to a fixed point of primitive Pisot substitution is that it remains within bounded distance from the expanding direction (given by the right eigenvector of Perron-Frobenius of $\mathrm{M}_{\sigma}$ ). We denote by $\mathrm{E}_{s}$ the stable space (or contracting space) and $\mathrm{E}_{u}$ the unstable space (or expanding direction). We denote by $\pi_{s}$ the linear projection in the contracting plane, parallel to the expanding direction and $\pi_{u}$ the projection in the expanding direction parallel to the contracting plane. We will project the stepped line on the contracting space in the direction of the right PerronFrobenius eigenvector. We obtain a bounded set in (d-1)dimensional vector space.

Definition 2.4. Let $\sigma$ an irreducible Pisot substitution. The Rauzy fractal associated to $\sigma$ is the closure of the projection of the canonical stepped line associated to any fixed point of $\sigma$ in the contracting plane parallel to the expanding direction.

We denote by $\mathrm{X}_{\sigma}$ the Rauzy fractal (Central tile) associated to $\sigma: \mathrm{X}_{\sigma}:=\overline{\{(\pi s \mathrm{l}(u 0 \ldots u \mathrm{k}-1), \mathrm{k} \in \mathbb{N}\}}$. with $u_{0} \ldots u_{\mathrm{k}-1}$ is a prefix of the fixed point of length $\mathrm{k}$. Subtiles of the central associated to the vertex of the stepped line that is projected. On thus sets for

i $\epsilon$ A: $\mathrm{X}_{\sigma}(\mathrm{i}):=\overline{\{\pi s(\mathrm{l}(\mathrm{u} 0 \ldots \mathrm{uk}-1), \mathrm{k} \in \mathbb{N}, \mathrm{uk}=\mathrm{l}\}}$.

\section{COMMON POINTS OF THE TRIBONACCI SUBSTITUTIONS}

\section{Common points of stepped lines}

In this part we will done some generalization of wok of Victor Sirvent in [19], where he done common dynamics of these two systems using the product automaton. With the product automaton, Victor Sirvent show realization in the plane and can define intersection in geometrical way, but he obtain a subset from the intersection of the Rauzy fractals associated to $\sigma_{1}$ and $\sigma_{2}$. This subset has a zero measure. in this part we will characterize all the intersection and we prove that it can be generated with a morphisim on the alphabet of balanced pair.

The idea is to consider the two stepped line associated to fixed points of $\sigma_{1}$ and $\sigma_{2}$, and we try to characterize the intersection of this two stepped lines.

Theorem 3.1. Let $\sigma_{1}$ and $\sigma_{2}$ be the two Tribonacci substitutions. We consider $L_{1}$ and $L_{2}$ the canonical broken lines associated to fixed points of $\sigma_{1}$ and $\sigma_{2}$ respectively. Let $P_{1}$ and $P_{2}$ be two points from $L_{1}$ and $L_{2}$ respectively. Then $\pi_{s}\left(P_{1}\right)=\pi_{s}\left(P_{2}\right)$ implies $P_{1}=P_{2}$.

Proof. We project $P_{1}$ and $P_{2}$ in the contracting space parallel to the direction of the Perron Frobenius eigenvectors, since The Tribonacci number is irrational, we can note obtain two points that they have the same projection.

From now we will consider the common points the two stepped lines of $L_{1}$ and $L_{2}$, and we will try to prove that these common points can be seen as a fixed point of a new morphism.

Definition 3.1. Let $U$ and $V$ be two finite words, we say that $\left(\begin{array}{l}U \\ V\end{array}\right)$ i abelianization map from $\boldsymbol{A}^{*}$ in $\mathbb{Z}^{\mathrm{d}}$.

Definition 3.2. A minimal balanced pair is a balanced pair, such for every strict prefix

$U_{\mathrm{k}}, V_{\mathrm{k}}$ of $U$ and $V$ respectively of length $\mathrm{k}, l\left(U_{\mathrm{k}}\right) \neq l\left(V_{\mathrm{k}}\right)$.

Theorem 3.2. Let $u$ and $v$ be two fixed points of the Tribonacci substitutions. We can decompose $u$ and $v$ with a finite number of minimal balanced pair. 


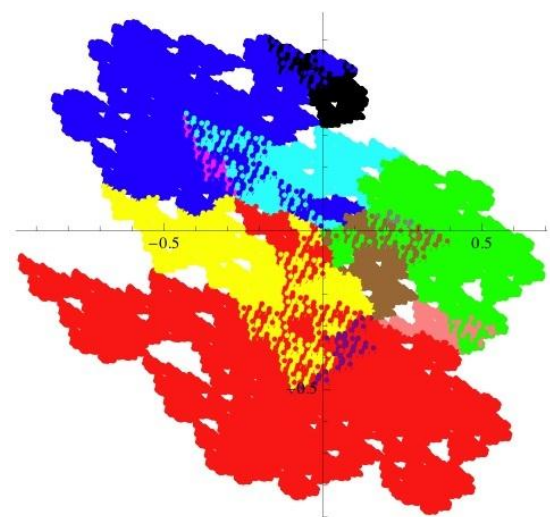

Figure 3: Sets of common points of the Tribonacci substitution and the flipped substitution. Each color stands for a different letter of $\boldsymbol{B}$ and shows the dynamics of the morphism $\phi$.

Proof. We have $u$ and $v$ are two fixed points of $\sigma_{1}$ and $\sigma_{2}$ then $u=$ abacabab ... and $v=$ abcaaababa... The first minimal balanced block is $A=\left(\begin{array}{l}a \\ a\end{array}\right)$. We consider the image of $A$ by

$\sigma_{1}$ and $\sigma_{2}$. we obtain $\left(\begin{array}{l}a \\ a\end{array}\right) \stackrel{\sigma 1, \sigma 2}{\longrightarrow}\left(\begin{array}{l}a b \\ b a\end{array}\right)$.

Now another minimal balanced pair appear, we denote $\mathrm{B}=\left(\begin{array}{l}b \\ b\end{array}\right)$ and we obtain the image of $A$ is $A B$.

We continues with this algorithm and we consider the image of each new minimal balanced pair.

Finally we obtain a finite number of balanced pairs, and we can define a morphism on these set of balanced pairs.

$$
\phi=\left\{\begin{array}{l}
\mathrm{A} \rightarrow \mathrm{AB} \\
\mathrm{B} \rightarrow \mathrm{C} \\
\mathrm{C} \rightarrow \mathrm{AD} \\
\mathrm{D} \rightarrow \mathrm{AE} \\
\mathrm{E} \rightarrow \mathrm{F} \\
\mathrm{F} \rightarrow \mathrm{ADDGA} \\
\mathrm{G} \rightarrow \mathrm{AH} \\
\mathrm{H} \rightarrow \mathrm{ID} \\
\mathrm{I} \rightarrow \mathrm{ADJ} \\
\mathrm{J} \rightarrow \mathrm{AHK} \\
\mathrm{K} \rightarrow \mathrm{IDGA}
\end{array}\right.
$$

and the projection map $\phi$ :

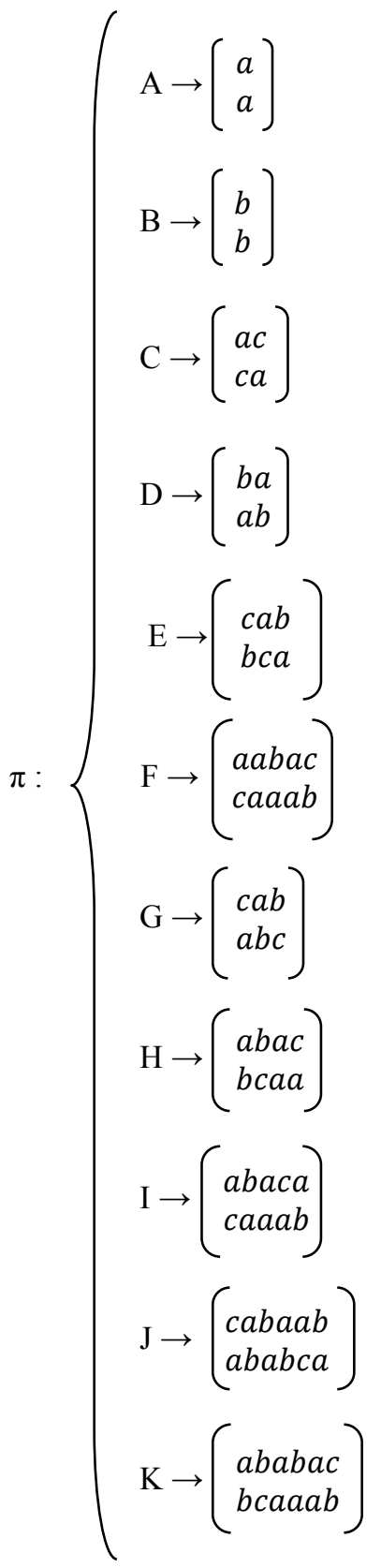

We obtain finally

$$
\left(\begin{array}{c}
\sigma_{1}{ }^{\mathrm{n}}(\mathrm{a}) \\
\sigma_{2}{ }^{\mathrm{n}}(\mathrm{a})
\end{array}\right)=\pi\left(\phi^{\mathrm{n}}(A)\right) .
$$

The morphism $\phi$ generate all the common points of the two stepped lines associated to $u$ and $v$.

Now we consider the stepped line associated of common points of $u$ and $v$, and we take the closure of the projection in 
the contracting plane parallel to the expanding direction and we obtain a Rauzy fractal associated to the intersection. See

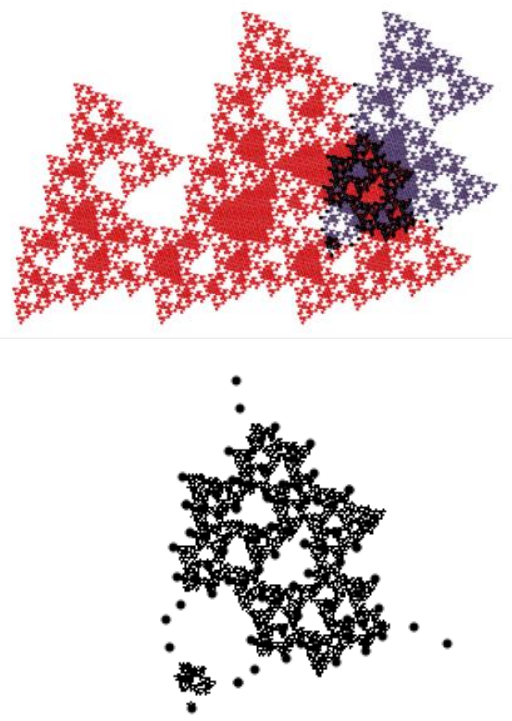

Figure 4: Sets of common points of intersection tiles A.
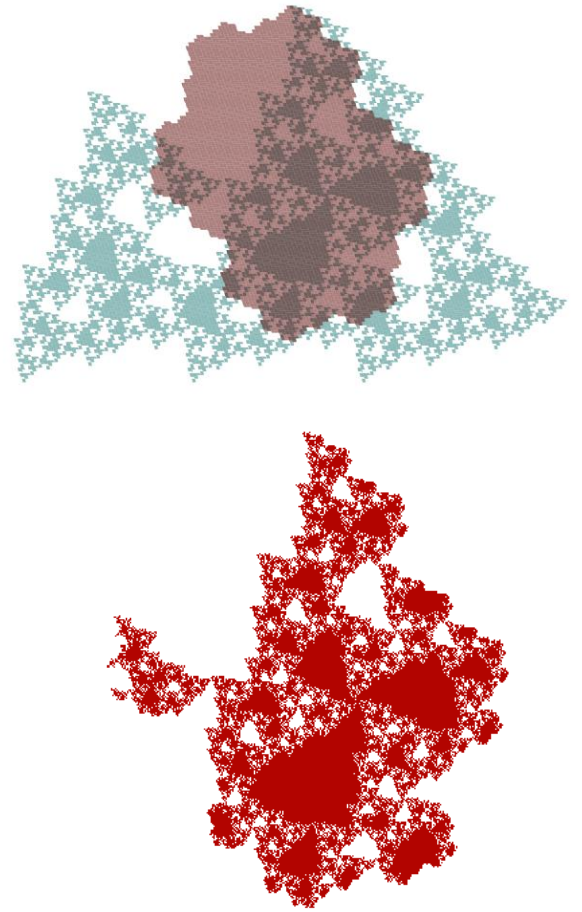

Figure 5: Sets of common points tile B.

\section{Remarks}

Corollaire 3.1. The letter $c$ doesn't occur in the same position in $u$ and $v$.

Proof. Minimal balanced blocks represents a decomposition of the two fixed points $u$ and $v$. We remark that in these finite minimal blocks there is no $c$ which appears in the same position. One can then deduce that the letter $c$ does not appear in the same position in two fixed points of $u$ and $v$.

Corollaire 3.2. Let $P_{\sigma}$ the characteristic polynomial of the incidence matrix of $\sigma_{1}$, and $P_{\phi}$ the characteristic polynomial of the incidence matrix of $\phi$.

Then $P_{\phi}=P_{\sigma}$. Q where $\mathrm{Q}$ is a polynomial of degree 9 .

\section{REFERENCES}

[1] P. Arnoux and S. Ito "Pisot substitutions and Rauzy fractals" Bull. Belg. Math. Soc. Simon Stevin 8: 181--207 (2001).

[2] M.Barge and B.Diamond. "Coincidence for substitutions of Pisot Type". Bell.Soc.Math. France, 130:619-626, 2002.

[3] Marcy Barge and Jarek Kwapisz, "Geometric theory of unimodular Pisot substitutions". American journal of mathematics(Print) 128:55, 1219-1282.

[4] Vicent Canterini and Anne Siegel : \{"Automate des préfixessuffixes associé une substitution primitive".J. Théor. Nombres Bordeaux 13, no. 2, 353--369, 2001.

[5] Amara Chandoul : \{"On the continued fraction of fixed period in finite filds", Canadian mathematical bulletin.\}.

[6] Amara Chandoul : \{"The Pell Equation", Advances in Pure Mathematics..

[7] Amara Chandoul : \{"On the continued fraction over the filds of formal power series", Canadian mathematical bulletin.

[8] Amara Chandoul : \{"On Polynomials solutions of quadratic Diophantine Equations.", Advances in Pure Mathematics.. \}

[9] F. M. Dekking, recurrent sets, Adv. in Math. 44 (1982), no. 1, 78-104. MR $84: 52023$.

[10] P. Fogg, "Substitutions in dynamics, arithmetics and combinatorics" (Lecture Botes in Mathematics, Vol. 1794).

[11] J. M. Luck, C. Godrèche and A. Janner and T. Janssen, "The nature of the atomic surfaces of quasiperiodic self similar structures", J. Phys. A : Math. Gen. 26 : 1951-1999 (1993).

[12] A. Messaoudi: "Propriétés arithmétiques et dynamiques du fractal de Rauzy", journal de Théorie des nombres de bordeaux, 10, 1998, 135-162.

[13] G. Rauzy: "Nombre algébrique et substitution", Bull.Soc.Math. France 110 (1982), 147-178.

[14] M. Queffelec, "Substitution dynamical system", Spectra analysis, lecture note in mathematics, 1294, Springer-Verlag, Berlin, 1987.

[15] Anne Siegel: "Autour des fractals de Rauzy", Journées Femmes et Mathématiques, Paris (03/2002).

[16] Anne Siegel and Jorg M.Thuswaldner: "Topological proprieties of Rauzy fractal", Mémoires de la SMF.

[17] Victor F.Sirvent: "The common dynamics of the tribonacci substitutions", Bull. Belg.Math..Soc. 7 (2000), 571-582.

[18] B. Sing and V. Sirvent: "Geometry of the common dynamics of flipped Pisot substitution", Monatshefte für Mathematik, 155 (2008), 431-448.

[19] Victor Sirvent and yang Sirvent, "Self affine tiling via substitution dynamical systems and Rauzy fractal". Pacific journal of mathematics Vol 206, N 2, 2002. 